\title{
LINC00844 promotes proliferation and migration of hepatocellular carcinoma by regulating NDRG1 expression
}

\author{
Wei Zhou ${ }^{1}$, Kang Huang ${ }^{1}$, Qiuyan Zhang ${ }^{1}$, Shaojun Ye ${ }^{1}$, Zibiao Zhong ${ }^{1}$, Cheng Zeng $^{1}$, Guizhu Peng $^{1}$, Ling Li $^{\text {Corresp., }}$ \\ 1 , Qifa Ye ${ }^{\text {Corresp. 1,2 }}$ \\ ${ }^{1}$ Zhongnan Hospital of Wuhan University, Institute of Hepatobiliary Diseases of Wuhan University, Transplant Center of Wuhan University, Hubei Key \\ Laboratory of Medical Technology on Transplantation, Wuhan, China \\ 2 The 3rd Xiangya Hospital of Central South University, Research Center of National Health Ministry on Transplantation Medicine Engineering and \\ Technology, Changsha, China \\ Corresponding Authors: Ling Li, Qifa Ye \\ Email address: Wb001019@whu.edu.cn,yqf_china@163.com
}

Background. Aberrant expression of long-noncoding RNAs is implicated int he pathogenesis of human malignancies. LINC00844 expression is dramatically downregulated in prostate cancer, and functional studies have revealed the association between the aberrant expression of LINC00844 and prostate cancer cell invasion and metastasis. However, the function and mechanism of action of LINC00844 in the pathogenesis of hepatocellular carcinoma (HCC) are poorly understood.

Methods. LINC00844 and N-Myc downstream-regulated 1 (NDRG1) expression in HCC tissues and cell lines was detected with real-time quantitative polymerase chain reaction (RT-qPCR) and western blot analysis. Correlations between LINC00844 expression level and clinicopathological features were investigated using the original data from The Cancer Genome Atlas (TCGA) database. HepG2 and HCCLM9 cell lines were transfected with Lv-LIN00844 virus to obtain LINC00844-overexpressing cell lines. Cell proliferation and cell invasion and migration were examined with the cell counting kit 8 (CCK8) and transwell assay, respectively. Furthermore, the correlation between LINC00844 and NDRG1 expression was analysed using Pearson's correlation analysis.

Results. LINC00844 expression was significantly downregulatedin HCC tissues and cell lines, and a statistical correlation was detected between low LINC00844 expression and sex (Female), advanced American Joint Committee on Cancer (AJCC) stage (III + IV), histological grade (G3 + G4), and vascular invasion (Micro and Macro).In vitro experiments showed that LINC00844 overexpression significantly repressed the proliferation, migration, and invasion of HCC cells. NDRG1 expression was higher in HCC tissues and LINC00844 could partly inhibit the expression of NDRG1. 
1 LINC00844 promotes proliferation and migration of hepatocellular carcinoma by

2 regulating NDRG1 expression

3 Wei Zhou ${ }^{1}$, Kang Huang ${ }^{1}$, Qiuyan Zhang ${ }^{1}$, Shaojun Ye$^{1}$, Zibao Zhong ${ }^{1}$, Cheng Zeng ${ }^{1}$, Guizhu

4 Peng ${ }^{1}$, Ling $\mathrm{Li}^{1}$, Qifa $\mathrm{Ye}^{1,2}$

5 1. Zhongnan Hospital of Wuhan University, Institute of Hepatobiliary Diseases of Wuhan

6 University, Transplant Center of Wuhan University, Hubei Key Laboratory of Medical

7 Technology on Transplantation, Wuhan, Hubei, China

8 2.The 3rd Xiangya Hospital of Central South University, Research Center of National Health

9 Ministry on Transplantation Medicine Engineering and Technology, Changsha, Hunan, China

10 Corresponding Author:

11 Qifa Ye,Wuhan, Hubei, 430071, China

12 Email address: yqf_china@163.com

13 Ling Li, Wuhan, Hubei, 430071, China

14 Email address: liling.12345@126.com

\section{Abstract}

\section{Background}

17 Aberrant expression of long-noncoding RNAs is implicated in the pathogenesis of human 18 malignancies. LINC00844 expression is dramatically downregulated in prostate cancer, and 19 functional studies have revealed the association between the aberrant expression of LINC00844 20 and prostate cancer cell invasion and metastasis. However, the function and mechanism of action 21 of LINC00844 in the pathogenesis of hepatocellular carcinoma (HCC) are poorly understood.

\section{Methods}


23 LINC00844 and N-Myc downstream-regulated 1 (NDRG1) expression in HCC tissues and cell

24 lines was detected with real-time quantitative polymerase chain reaction (RT-qPCR) and western

25 blot analysis. Correlations between LINC00844 expression level and clinicopathological features

26 were investigated using the original data from The Cancer Genome Atlas (TCGA) database.

27 HepG2 and HCCLM9 cell lines were transfected with Lv-LIN00844 virus to obtain LINC00844-

28 overexpressing cell lines. Cell proliferation and cell invasion and migration were examined with

29 the cell counting kit 8 (CCK8) and transwell assay, respectively. Furthermore, the correlation

30 between LINC00844 and NDRG1 expression was analysed using Pearson's correlation analysis.

\section{Results}

LINC00844 expression was significantly downregulated in HCC tissues and cell lines, and a statistical correlation was detected between low LINC00844 expression and sex (Female), advanced American Joint Committee on Cancer (AJCC) stage (III + IV), histological grade (G3 + G4), and vascular invasion (Micro and Macro). In vitro experiments showed that LINC00844 overexpression significantly repressed the proliferation, migration, and invasion of HCC cells. NDRG1 expression was higher in HCC tissues and LINC00844 could partly inhibit the expression of NDRG1.

Introduction

Hepatocellular carcinoma (HCC) is a common primary liver tumour and one of the most common causes of cancer-related deaths worldwide ${ }^{[1]}$. Hepatitis B virus (HBV) infection, chronic Hepatitis C virus (HCV) infection, alcohol abuse, aflatoxin B1 intake, and metabolic syndrome are high risk factors associated with liver cancer. The incidence of HBV infection is the highest in Asia ${ }^{[2]}$. There is still no cure for chronic HBV infection ${ }^{[3]}$. Despite tremendous progress in HCC therapy, an effective strategy to cure HCC is still missing ${ }^{[2]}$. As observed with many other cancers, HCC tumourigenesis and progression is a complex process involving genetic and epigenetic transformations in pivotal growth regulatory genes ${ }^{[4,5]}$. Therefore, it is 
48 important to clearly elucidate the molecular mechanism underlying the occurrence of HCC and 49 develop novel strategies for the early diagnosis, prognosis, and treatment of patients with HCC.

50 Evidence suggests that long-noncoding RNAs (lncRNAs) play an important role in the regulation 51 of several biological processes related to hepatocarcinogenesis, including cell proliferation, 52 apoptosis, metastasis, angiogenesis, and chemosensitivity ${ }^{[6,7,8,9]}$. Many lncRNAs play crucial 53 roles in the oncogenesis and development of malignant tumours and function as oncogenes or 54 tumour suppressors ${ }^{[10]}$. LINC00844 is a 659 bp lncRNA transcribed from chromosome 55 chr10q21.1 and comprises two exons ${ }^{[11,12]}$. N-myc downstream-regulated gene 1 (NDRG1) 56 belongs to an NDR- and an $\alpha / \beta$ hydrolase-fold region family, and is known to promote 57 adipogenesis and sustain adipocyte function by inducing peroxisome proliferator-activated 58 receptor gamma (PPAR $\gamma$ ) expression and $\mathrm{C} / \mathrm{Ebp} \alpha$ activity under physiological conditions ${ }^{[13]}$. In 59 differentiated normal epithelial cells, NDRG1 retains the stability of tight junctions by regulating the expression of claudin-9[14]. However, NDRG1 expression was found to be upregulated in patients with HCC as compared with that in healthy controls and correlated with poorer outcomes $^{[15]}$. NDRG1 expression may be influenced by many factors, especially hypoxiainducible factor-1 (HIF-1) ${ }^{[16]}$. Previous studies have shown that NDRG1 is involved in tumour invasion and metastasis ${ }^{[17]}$. A study reported that NDRG1 overexpression may inhibit the expression of E-cadherin and enhance the expression of Snail, and consequently regulate tumour growth and metastasis in oesophageal squamous cell carcinoma ${ }^{[18]}$. Another study reported that LINC00844 exerted its antitumour activity by regulating the expression of NDRG1 in prostate cancer $^{[19]}$. However, the biological function and underlying molecular mechanisms of LINC00844 in HCC are unclear.

Materials \& Methods

\section{Gene expression profiles}

73 We downloaded the gene expression profiling data from The Cancer Genome Atlas database 74 (TCGA, http://portal.gdc.cancer.gov/), which is available for free. A total of 424 tissue samples comprising 371 primary HCC samples, 3 recurrent HCC samples, and 50 normal liver tissue 
77 based on the R language and $t$-test were used to evaluate differentially expressed genes.

78 Cell culture

79 The human HCC cell lines (HCCLM9, SMMC-7721, SK-Hep1, and HepG2) and a normal 80 hepatic epithelial cell line (L02) were purchased from the Cell Bank of Type Culture Collection 81 (CBTCC, Chinese academy of sciences, Shanghai, China), and these cell lines were cultured in 82 Dulbecco's modified Eagle's medium (DMEM; Invitrogen Life Technology Inc., Carlsbad, CA) 83 supplemented with 10\% foetal bovine serum (FBS; Gibco, Grand Island, NY, USA), $100 \mu \mathrm{g} / \mathrm{mL}$ 84 streptomycin, and $100 \mathrm{U} / \mathrm{mL}$ penicillin at $37^{\circ} \mathrm{C}$ in a $5 \% \mathrm{CO}_{2}$ incubator.

85 Tissue specimens

86 Forty HCC tissues and paired adjacent non-tumour tissues were collected from the Zhongnan 87 Hospital of Wuhan University from April 2018 to April 2019. Surgically resected tissue samples 88 were immediately frozen in RNAlater (Qiagen, Hilden, Germany) and subsequently stored at $89-80^{\circ} \mathrm{C}$ until use. The patients provided signed informed consent, and the study was approved by 90 the Medical Ethics Committee of Zhongnan Hospital of Wuhan University. The approval 91 number is 2019016.

\section{Plasmid construction, lentiviral packaging, and cell transfection}

93 Total RNA was isolated from HCC tissues or cells by TRIZOL Reagent (Yeasen Biotech, 94 Shanghai, China) following the manufacturer's instructions. After extraction, RNA samples were 95 reverse transcribed by a reverse transcription kit (Yeasen Biotech, Shanghai, China) and its 96 concentration was determined using a NanoDrop ND-1000 spectrophotometer (Thermo Fisher 97 Scientific, Wilmington, DE). The Hieff qPCR SYBR Green Master Mix kit (Yeasen Biotech, 98 Shanghai, China) was used for real-time quantitative polymerase chain reaction (RT-PCR). 99 Expression levels were normalised against glyceraldehyde-3-phosphate dehydrogenase 100 (GAPDH) level, and the $2^{-\Delta \Delta C T}$ method was used to calculate relative fold change. All primers 101 were designed and synthesised by Wuhan Servicebio Technology CO., LTD (Wuhan, China) and 102 their sequences are listed in Table 1.

103 RT-qPCR 
104 Full-length LINC00844 was amplified and cloned into a lentiviral expression vector pEZ-Lv201

105 (Generay Biotech Co. Ltd, Shanghai, China) and subjected to lentiviral packaging (Generay

106 Biotech Co. Ltd, Shanghai, China). Cell lines were infected with Lv-LINC00844 viruses, while

107 Lv-NC viruses served as the negative control. Efficiency was confirmed with RT-qPCR.

108 Transwell assay

109 Transwell assay was used to detect the migration and invasion abilities of cells. A 24-well

110 transwell chamber without or with matrigel (Corning Life Sciences, Corning, NY, USA) was

111 used to detect cell migration and invasion rates. In brief, a $100 \mu \mathrm{L}$ cell suspension

$112\left(5 \times 10^{4}\right.$ cells/well $)$ in a serum-free medium was seeded into the upper chamber, while the lower 113 chamber was filled with $600 \mu \mathrm{L}$ of DEME containing $10 \%$ FBS. Cells were incubated on the

114 membranes for $24 \mathrm{~h}$, followed by fixation of the migrated cells in $4 \%$ paraformaldehyde for 20

115 min and staining with $0.1 \%$ crystal violet in $1 \times$ phosphate-buffered saline (PBS) for $30 \mathrm{~min}$.

116 Cells were counted from five random fields under a microscope, and the average number of cells

117 per field was determined. All experiments were performed in triplicates.

\section{Cell proliferation assay}

119 Cell counting kit-8 (CCK-8, Yeasen Biotech, Shanghai, China) was used to detect cell 120 proliferation. Cells were plated in 96-well plates in triplicates at $2 \times 10^{3}$ cells/well and incubated 121 in a $5 \% \mathrm{CO}_{2}$ atmosphere at $37^{\circ} \mathrm{C}$. The absorbance of each well at $450 \mathrm{~nm}$ wavelength was 122 measured every $24 \mathrm{~h}$ as per the instructions of the manufacturer for 4 consecutive days obtain a 123 growth curve. All experiments were performed in triplicates.

\section{Immunohistochemistry (IHC)}

125 Tumour tissues were fixed in 4\% paraformaldehyde, dehydrated, embedded in paraffin, and cut 126 into 4- $\mu$ m-thick sections. Briefly, sections were dewaxed in xylene and rehydrated through 127 graded alcohol solutions. Endogenous peroxidase activity was quenched with hydrogen 128 peroxide and renovated antigen. The slides were incubated overnight with $10 \%$ serum to block 129 any nonspecific binding sites. Slides were then treated with anti-NDRG1 antibody (1:400 
130 dilution, Wuhan Proteintech Group, China) at $4^{\circ} \mathrm{C}$. The sections were eventually incubated with 131 a peroxidase-conjugated polymer for $30 \mathrm{~min}$, and a 3,3'-diaminobenzidine (DAB) system 132 (Beyotime Institute of Biotechnology, Jiangsu, China) was used for detection.

\section{Western blot analysis}

134 Total protein was extracted from HepG2 and HCCLM9 cells using radioimmunoprecipitation 135 assay (RIPA) lysis buffer (Wuhan Servicebio Biotechnology, China), and the protein 136 concentration was measured with a bicinchoninic acid (BCA) assay kit (Wuhan Google 137 Biotechnology Co., Ltd). Equal amounts of protein lysates were separated by sodium dodecyl 138 sulphate polyacrylamide gel electrophoresis (SDS-PAGE; Wuhan Google Biotechnology Co., 139 Ltd, China) and the separated bands were transferred onto nitrocellulose membranes. The 140 membranes were incubated overnight at $4^{\circ} \mathrm{C}$ with a primary rabbit anti-NDRG1 antibody $(1: 800$,

141 Wuhan Proteintech Group, China). Proteins were detected using an enhanced 142 chemiluminescence (ECL) method as previously described. GAPDH was used as a loading 143 control.

\section{Statistical analysis}

145 All data are presented as means \pm standard error of the mean (SEM). The Kaplan-Meier 146 method was used for survival analysis. Correlation between LINC00844 expression and 147 clinicopathological variables was analysed with the Student's $t$-test or one-way analysis of 148 variance (analysis of variance [ANOVA], $t$-test for two-group comparisons or one-way ANOVA 149 for other cases). Statistical analysis was performed using GraphPad Prism 7 and SPSS 18.0. $P<$ 1500.05 was considered statistically significant.

\section{Results}

152 Expression of LINC00844 is significantly downregulated in HCC tissues and cells

153 The top five upregulated lncRNAs and top five downregulated lncRNAs (adjusted $P$ value $<$ 1540.05 and $|\operatorname{logFC}| \geq 2$ ) are shown in the heat map, and included LINC00844 (Ensemble 
155 ID:ENSG00000237949, Fig. 1A and Table 2). To understand the expression pattern of 156 LINC00844 in different tumours, we extracted LINC00844 from all 31 tumour samples from 157 Gene Expression Profiling Interactive Analysis (GEPIA) (http://gepia.cancer-pku.cn/) and found 158 that LINC00844 expression was low in most tumours, including HCC (Fig. 1B). LINC00844 159 was downregulated in liver HCC (LIHC) from GEPIA (Fig. 2A). In our study, the relative 160 expression level of LINC00844 was detected in HCC tissues $(n=40)$ and their adjacent non161 tumour tissues $(n=40)$ with RT-qPCR. The relative expression level of LINC00844 was 162 significantly downregulated in HCC tissues as compared with that in paired adjacent non-tumour 163 tissues. LINC00844 expression downregulation ( $>$ twofold change) was detected in 87.5\% $164(35 / 40)$ of HCC tissues (Fig. 2F and Fig. 2G, $P<0.01$ ). The relative expression level of 165 LINC00844 correlated with histological grade from GEPIA (Fig. 2B, $P=0.0377$ ). However, 166 GEPIA showed that patients with low LINC00844 expression had no obvious association with 167 overall survival and disease-free survival (Fig. 2C and Fig. 2D). Moreover, the area under curve 168 (AUC) of LINC00844 expression was 0.854 (Fig. 2E, 95\% confidence interval [CI]: 0.805-0.886, $169 P<0.05)$. Thus, LINC00844 has a moderate diagnostic value in HCC.

170 Correlation between LINC00844 expression downregulation and clinicopathological 171 features of patients with HCC

172 To evaluate the clinical significance of LINC00844 in HCC, we explored the correlation between 173 LINC00844 expression and the clinicopathological features of patients with HCC. The results 174 are shown in Table 3. A statistically significant correlation was detected between LINC00844 175 expression level and sex (Fig. 3A, Male versus Female, $P<0.01$ ), histological grade (Fig. 3C, $176 \mathrm{G} 1+\mathrm{G} 2$ versus G3 + G4, $P<0.01$ ), advanced American Joint Committee on Cancer (AJCC) 177 stage (Fig. 3E, I + II versus III + IV, $P<0.05$ ), and vascular invasion (Fig. 3F, None versus 178 Micro versus Macro, $P<0.01)$. However, no significant association was observed between 179 LINC00844 expression and ethnicity (Fig. 3B, Asian versus White, $P>0.05$ ) and T 180 classification of TNM stage (Fig. 3D, T1 + T2 versus T3 + T4, $P>0.05$ ). The results are based 181 on the original data from TCGA. Meanwhile, To evaluate the clinical roles of LINC00844 in 
182 HCC, we also explored the correlation between LINC00844 expression and the 183 clinicopathological factors with $40 \mathrm{HCC}$ patients, and results was shown in Table4. Lower 184 expression of LINC00844 was closely correlated with multiple clinical pathological features 185 including pathological stage $(P<0.0484)$, portal vein tumor thrombus $(P=0.0471)$ and TNM $186(P<0.0187)$.

187 LINC00844 expression upregulation inhibits HepG2 and HCCLM9 cell proliferation, 188 migration, and invasion

189 We observed that HCC cell lines (HepG2, HCCLM9, SMMC-7721, and SK-Hep1) expressed 190 significantly lower levels of LINC00844 than the normal hepatic epithelial cell line L02 (Fig. 4A, $191 P<0.01)$. LINC00844 expression was relatively lower in HepG2 and HCCLM9 cell lines than 192 in SK-Hep1 and SMMC-7721 cell lines. Therefore, we chose HepG2 and HCCLM9 cell lines 193 for the following gain-of-function studies. Relative LINC00844 expression in HepG2 and 194 HCCLM9 cell lines was significantly increased upon infection with Lv-LINC00844; Lv-NC 195 viruses acted as a negative control (Fig. 4B, $P<0.01$ ). As per the results of the CCK-8 assay, the 196 OD450 values of HepG2 and HCCLM9 cell lines from Lv-LINC00844 group were remarkably 197 lower than those for the cells from Lv-NC group at 0, 24, 48, and $72 \mathrm{~h}$ (Fig.4C and Fig.4D, $P<$ 198 0.01). Cell migration and invasion abilities were analysed with transwell assays and found to be 199 significantly decreased for HepG2 and HCCLM9 cell lines from Lv-LINC00844 group as 200 compared with those from Lv-NC group (Fig.4E, Fig.4F, Fig.4G and Fig.4H, $P<0.01$ ).

201 NDRG1 is overexpressed in clinical HCC tissues and cell lines and negatively correlates 202 with LINC00844

203 To investigate the mechanism of action of LINC00844 in HCC tumourigenesis, we analysed the 204 mRNA level of NDRG1 in HCC tissues and cells and investigated the relationship between 205 NDRG1 and LINC00844 expression. NDRG1 expression was upregulated in LIHC from GEPIA 206 (Fig. 5A). We also examined the expression level of NDRG1 mRNA with RT-qPCR and found 207 that NDRG1 level was markedly elevated in $20 \mathrm{HCC}$ tissues as compared with that in paired 208 adjacent non-tumour tissues (Fig. 5D, $P<0.01$ ). The expression level of NDRG1 correlated with 
209 histological grade from GEPIA (Fig. 5B, $P=0.0126$ ). GEPIA demonstrated that high NDRG1 210 expression level showed a significant association with overall survival in patients with HCC (Fig. 211 5C). The expression level of LINC00844 was negatively associated with that of NDRG1 in 20 212 paired clinical HCC tissues (Fig. 5E, $P<0.05, \mathrm{R}^{2}=0.3043$, Pearson's correlation). IHC 213 assay was performed to investigate the expression of NDRG1 protein in HCC tissues (Fig. 5F). 214 An increase in LINC00844 expression resulted in a decrease in the mRNA and protein levels of 215 NDRG1 in HepG2 and HCCLM9 cells (Fig. 5G).

\section{Discussion}

217 Genome-wide sequencing has revealed the involvement of more and more lncRNAs in different 218 types of cancers ${ }^{[10]}$. Lingadahalli et al. ${ }^{[19]}$ performed in vivo and in vitro experiments to confirm 219 the participation of LINC00844 in tumour metastasis partly through its effect on the expression 220 of NDRG1 in prostate cancer. High expression of lncPARP1 may serve as an adverse prognosis 221 factor for $\mathrm{HCC}^{[20]}$. In our study, we used TCGA and GEPIA and found that the expression level 222 of LINC00844 was lower in HCC tissues than in normal samples. We also verified the dramatic 223 decrease in LINC00844 expression in 40 paired primary HCC tissues and cell lines as compared 224 with that in the paired adjacent non-tumour tissues and normal liver cell lines. We obtained data 225 on LINC00844 expression and relative clinical features from TCGA and found that low 226 LINC00844 expression was highly associated with late clinical stage, poor histological grade, 227 and vascular invasion in patients with HCC. And we discovered lower expression of LINC00844 228 was positively correlated with pathological stage, portal vein tumor thrombus and TNM in 40 229 HCC patients. These findings were the approximate homology with TCGA dataset. These results 230 suggest LINC00844 may have a clinical significance in the diagnosis of HCC. To investigate the 231 biological role of LINC00844 in HCC, LINC00844 expression was induced in HCC cells 232 (HepG2 and HCCLM9). As a result, LINC00844 overexpression was shown to significantly 233 inhibit proliferation and suppress migration and invasion of HepG2 and HCCLM9 cell lines. 234 Based on these results, we suggest that LINC00844 may act as a tumour suppressor in HCC.

235 As a key regulator, lncRNAs are involved in the regulation of gene expression in cancers, and 
236 play an important role in chromatin remodelling complexes and gene regulation at transcriptional 237 and transcriptional levels ${ }^{[21,22]}$. For instance, the lncRNA PCAT6 was shown to inhibit non-small 238 cell lung cancer cell proliferation, apoptosis, and metastasis through epigenetic mechanisms ${ }^{[23]}$. 239 MIR31HG suppressed HCC proliferation and metastasis by serving as an miR-575 sponge in 240 vitro and in vivo ${ }^{[24]}$. The lncRNA Xist is known to increase histone trimethylation level and 241 reduce the activity of $\mathrm{X}$ chromosome ${ }^{[25]}$. A recent report showed that LINC00844 inhibited 242 tumour metastasis by regulating the expression of NDRG1 in prostate cancer ${ }^{[17]}$. Growing 243 evidence has highlighted the regulatory role of NDRG1 in a variety of functions, including 244 cellular differentiation, activation of p53, and cellular apoptosis and metastasis ${ }^{[26,27,28]}$. Yan et 245 al. ${ }^{[29]}$ found that NDRG1 expression is generally upregulated in HCC tissues as compared with 246 that in normal samples, particularly in recurrent and metastatic HCC. NDRG1 overexpression 247 enhances aerobic glycolysis and imparts growth advantages to cells, induces the expression of 248 hypoxia-associated genes, and causes lipid metabolism dysfunction in breast cancer ${ }^{[30]}$. A report 249 revealed the role of the lncRNA CCAT2 in promoting cellular proliferation and metastasis 250 through the upregulation of NDRG1 expression in $\mathrm{HCC}^{[31]}$. Consistent with these results, we 251 confirm that NDRG1 expression was upregulated in 20 pairs of HCC tissues and that patients 252 with higher NDRG1 expression showed poorer clinical prognosis and histological grade. The 253 expression of NDRG1 mRNA was negatively correlated with LINC00844 in HCC tissues, and 254 LINC00844 overexpression could partly inhibit the expression of NDRG1 in HepG2 and 255 HCCLM9 cells. How LINC00844 controls tumour migration and invasion in HCC warrants 256 further studies.

\section{Conclusions}

258

In summary, we demonstrated that the expression of LINC00844 is downregulated in HCC samples and cell lines. LINC00844 overexpression inhibits HCC cell proliferation and invasion, at least in part, through the suppression of NDRG1 expression. Thus, LINC00844 may be potentially used as a promising prognostic biomarker and therapeutic target for HCC. 


\section{Acknowledgements}

263 We would like to thank all tutors and friends.

\section{Abbreviations}

265 lncRNA, long-noncoding RNA

266 LINC00844, long intergenic non-protein coding RNA 844

267 HCC, hepatocellular carcinoma

268 NDRG1, N-myc downstream-regulated gene 1

269 RT-qPCR, real-time quantitative polymerase chain reaction

270 TCGA, The Cancer Genome Atlas

271 CCK8, cell counting kit-8

272 AJCC, American Joint Committee on Cancer

273 HBV, hepatitis B virus

$274 \mathrm{HCV}$, hepatitis $\mathrm{C}$ virus

275 HIF-1, hypoxia-inducible factor 1

276 DMEM, Dulbecco's modified Eagle's medium

277 GAPDH, glyceraldehyde-3-phosphate dehydrogenase

278 PBS, phosphate-buffered saline

279 IHC, immunohistochemistry

280 DAB, 3, 3'-diaminobenzidine

281 RIPA, radioimmunoprecipitation assay

282 SDS, sodium dodecyl sulphate

283 PAGE, polyacrylamide gel electrophoresis

284 LIHC, liver hepatocellular carcinoma

285 GEPIA, gene expression profiling interactive analysis

286 AUC, area under curve

287 OD450, optical density $405 \mathrm{~nm}$

\section{References}


289 1.Bray F, Ferlay J, Soerjomataram I, Siegel RL, Torre LA, Jemal A. 2018. Global cancer

290 statistics 2018: Globocan estimates of incidence and mortality worldwide for 36 cancers in 185

291 countries. CA Cancer J Clin 68:394-424.

292 2.Forner A, Reig M, Bruix J. 2018. Hepatocellular carcinoma. Lancet 391:1301-1314.

293 3.Ji M, Hu K. 2017. Recent advances in the study of hepatitis B virus covalently closed circular 294 DNA. Virol Sin 32:454-464.

295 4.Furuta M, Kozaki KI, Tanaka S, Arii S, Imoto I, Inazawa J. 2010. miR-124 and miR-203 are

296 epigenetically silenced tumor-suppressive microRNAs in hepatocellular carcinoma.

297 Carcinogenesis 31:766-776.

298 5.Forner A, Llovet JM, Bruix J. 2012. Hepatocellular carcinoma. Lancet 379:1245-1255.

299 6.Lin J, Cao S, Wang Y, Hu Y, Liu H, Li J, Chen J, Li P, Liu J, Wang Q, Zheng L. 2018. Long

300 non-coding RNA UBE2CP3 enhances HCC cell secretion of VEGFA and promotes angiogenesis

301 by activating ERK1/2/HIF-1 $\alpha$ /VEGFA signalling in hepatocellular carcinoma. J Exp Clin Cancer 302 Res 37:117.

303 7.Deng L, Yang SB, Xu FF, Zhang JH. 2015. Long noncoding RNA CCAT1 promotes

304 hepatocellular carcinoma progression by functioning as let-7 sponge. J Exp Clin Cancer Res $30534: 18$.

306 8.Runzhou Zhuang, Xuanyu Zhang, Di Lu, Jianguo Wang, Jianyong Zhuo, Xuyong Wei, Qi Ling, 307 Haiyang Xie, Shusen Zheng, Xiao Xu. 2019. LncRNA DRHC inhibits proliferation and invasion 308 in hepatocellular carcinoma via c-Myb-regulated MEK/ERK signaling. Mol Carcinog 58:366309375.

310 9.Yi Zhang, Bo Tang, Jun Song, Shuiping Yu, Yang Li, Huizhao Su, Songqing He. 2019. Lnc311 PDZD7 contributes to stemness properties and chemosensitivity in hepatocellular carcinoma 312 through EZH2-mediated ATOH8 transcriptional repression. J Exp Clin Cancer Res 38:9.

313 10.Bach DH, Lee SK. 2018. Long noncoding RNAs in cancer cells. Cancer Lett 419:152-166. 314 11.Wang AG, Yoon SY, Oh JH, Jeon YJ, Kim M, Kim JM, Byun SS, Yang JO, Kim JH, Kim 315 DG, Yeom YI, Yoo HS, Kim YS, Kim NS. 2006. Identification of intrahepatic 
316 cholangiocarcinoma related genes by comparison with normal liver tissues using expressed 317 sequence tags. Biochem Biophys Res Commun 345:1022-1032.

318 12.KimuraK, Wakamatsu A, Suzuki Y, Ota T, Nishikawa T, Yamashita R, Yamamoto J, Sekine

319 M, Tsuritani K, Wakaguri H, Ishii S, Sugiyama T, Saito K, Isono Y, Irie R, Kushida N,

320 Yoneyama T, Otsuka R, Kanda K, Yokoi T, Kondo H, Wagatsuma M, Murakawa K, Ishida S,

321 Ishibashi T, Takahashi-Fujii A, Tanase T, Nagai K, Kikuchi H, Nakai K, Isogai T, Sugano S.

322 2006. Diversification of transcriptional modulation: large-scale identification and

323 characterization of putative alternative promoters of human genes. Genome Res 16:55-65.

324 13.Cai K, EI-Merahbi R, Loeffler M, Mayer AE and Sumara G. 2017. Ndrg1 promotes adipocyte 325 differentiation and sustains their function. Sci Rep.7(1):7191.

326 14.Gao Y, Maruoka S, Kishi H, Kozu Y, Kazumichi K, Nomura Y, Takeshita I, Oshima T and 327 Hashimoto S. 2017. NDRG1 is important to maintain the integrity of airway epithelial barrier 328 through claudin-9 expression. Cell Biol Int. 41(7):716-725

329 15.Cheng J, Xie HY, Xu X, Wu J, Wei X, Su R, Zhang W, Lv Z, Zhang S, Zhou L. 2011. 330 NDRG1 as a biomarker for metastasis, recurrence and of poor prognosis in hepatocellular 331 carcinoma. Cancer Lett 310:35-45.

332 16.Salnikow K, Kluz T, Costa M, Piquemal D, Demidenko ZN, Xie K, Blagosklonny MV. 2002. 333 The regulation of hypoxic genes by calcium involves c-Jun/AP-1, which cooperates with 334 hypoxia-inducible factor 1 in response to hypoxia. Mol Cell Biol 22:1734-1741.

335 17.Li A, Zhu X, Wang C, Yang S, Qiao Y, Qiao R, Zhang J. 2019. Upregulation of NDRG1 336 predicts poor outcome and facilitates disease progression by influencing the EMT process in 337 bladder cancer. Sci Rep 9:5166.

338 18.Ai R, Sun Y, Guo Z, Wei W, Zhou L, Liu F, Hendricks DT, Xu Y and Zhao X. 2016. NDRG1 339 overexpression promotes the progression of esophageal squamous cell carcinoma through 340 modulating Wnt signaling pathway. Cancer Biol Ther. 17(9):943-954.

341 19.Lingadalli S, Jadhao S, Sung YY, Chen M, Hu L, Chen X, Cheung E. 2018. Novel

342 IncRNA LINC00844 Regulates Prostate Cancer Cell Migration and Invasion through AR 
343 Signaling. Mol Cancer Res 16:1865-1878.

344 20.Qi H, Lu Y, Lv J, Wu H, Lu J, Zhang C, Zhang S, Bao Q, Zhang X, Xie C, Yin Z. 2018.

345 The long noncoding RNA lncPARP1 contributes to progression of hepatocellular carcinoma

346 through up-regulation of PARP1. Biosci Rep 38: BSR20180703.

347 21.Schmitt AM, Chang HY. 2016. Long noncoding RNAs in cancer pathways. Cancer Cell $348 \quad 29: 452-463$.

349 22.Quinn JJ, Chang HY. 2016. Unique features of long non-coding RNA biogenesis and function. 350 Nat Rev Genet 17:47-62.

351 23.Shi X, Liu Z, Liu Z, Feng X, Hua F, Hu X, Wang B, Lu K, Nie F. 2018. Long noncoding 352 RNA PCAT6 functions as an oncogene by binding to EZH2 and suppressing LATS2 in non353 small-cell lung cancer. EBioMedicine 37:177-187.

354 24.Yan S, Tang Z, Chen K, Liu Y, Yu G, Chen Q, Dang H, Chen F, Ling J, Zhu L, Huang A, 355 Tang H. 2018. Long noncoding RNA MIR31HG inhibits hepatocellular carcinoma proliferation 356 and metastasis by sponging microRNA-575 to modulate ST7L expression. J Exp Clin Cancer 357 Res 37:214.

358 25.Simon MD, Pinter SF, Fang R, Sarma K, Rutenberg-Schoenberg M, Bowman SK, Kesner BA, 359 Maier VK, Kingston RE, Lee JT. 2013. High-resolution Xist binding maps reveal two-step 360 spreading during X-chromosome inactivationNature 504:465-469.

361 26.Sibold S, Roh V, Keogh A, Studer P, Tiffon C, Angst E, Vorburger SA, Weimann R, 362 Candinas D, Stroka D. 2007. Hypoxia increases cytoplasmic expression of NDRG1, but is 363 insufficient for its membrane localization in human hepatocellular carcinoma. FEBS 364 Lett 581:989-994.

365 27.Byun JW, An HY, Yeom SD, Lee SJ, Chung HY. 2018. NDRG1 and FOXO1 regulate 366 endothelial cell proliferation in infantile haemangioma. Exp Dermatol 27:690-693.

367 28.Chen B, Zaveri PG, Longtine MS, Nelson DM. 2015. N-myc downstream-regulated gene 1 368 (NDRG1) mediates pomegranate juice protection from apoptosis in hypoxic BeWo cells but not 369 in primary human trophoblasts. Placenta 36:847-853. 
370

371

372

373

374

375

376

377

378

379

380

381

382

383

384

385

386

387

388

389

390

391

392

393

394

395

396

29. Yan X, Chua MS, Sun H, So S. 2008. N-Myc down-regulated gene 1 mediates proliferation, invasion, and apoptosis of hepatocellular carcinoma cells. Cancer Lett 262:133-142.

30.Sevinsky CJ, Khan F, Kokabee L, Darehshouri A, Maddipati KR, Conklin DS. 2018. NDRG1

regulates neutral lipid metabolism in breast cancer cells. Breast Cancer Res 20:55.

31.Liu Y, Wang D, Li Y, Yan S, Dang H, Yue H, Ling J, Chen F, Zhao Y, Guo L, Tang P, Huang A, Tang H. 2019. Long noncoding RNA CCAT2 promotes hepatocellular carcinoma proliferation and metastasis through up-regulation of NDRG1. Exp Cell Res 379:19-29.

\section{FIGURE LEGENDS}

Figure 1: Analysis of the expression of LINC00844 in TCGA and GEPIA databases.

(A) Heat map of the top five upregulated and top five downregulated lncRNAs.

(B) Overview of LINC00844 expression in multiple tumour entities with a notable decrease in tumours as compared with that in normal tissues. The image is derived from GEPIA.

Figure 2: LINC00844 is significantly downregulated in HCC.

(A) Expression of LINC00844 was analysed in HCC from GEPIA. ${ }^{*} P<0.01$. (B) The relationship between the expression of LINC00844 and HCC pathological stage from GEPIA. *P $<0.05$. (C and D) Kaplan-Meier analysis of the correlation between LINC00844 expression and disease-free survival and overall survival in GEPIA. $P>0.05$. (E) ROC curve of LINC00844 in HCC derived from the original data from TCGA database. $P<0.01, \mathrm{AUC}=0.854$. (F and G) Expression and fold change of LINC00844 in 40 pairs of HCC tissues and adjacent non-tumour tissues. Downregulation of LINC00844 ( $>$ twofold change) expression was detected in 87.5\% $(35 / 40)$ of HCC. $* * P<0.01$. GAPDH was used as control. All results are obtained from at least three independent experiments.

Figure 3: Correlation between LINC00844 downregulation and clinicopathologic features in 254 HCC samples and 50 normal liver tissues based on the original data from TCGA.

(A, C, E, and F) The relationship between LINC00844 expression and sex (Male versus Female, $P<0.001)$, histological grade (G1 + G2 versus G3 + G4, $P<0.001)$, advanced American Joint Committee on Cancer (AJCC) stage (I + II versus III + IV, $P=0.0399$ ), and vascular invasion 
397 (None versus Micro versus Macro, $P=0.006$ ). (B and D) The relationship between the 398 expression of LINC00844 and ethnicity (Asian versus White, $P=0.5361$ ) and T classification of

399 TNM stage $(\mathrm{T} 1+\mathrm{T} 2$ versus T3 $+\mathrm{T} 4, P=0.1366)$.

400 Figure 4: Upregulation in LINC00844 expression represses the proliferation, migration, 401 and invasion of HepG2 and HCCLM9 cells.

402 (A) The relative expression of LINC00844 in HCC cell lines (HepG2, SK-Hep1, HCCLM9, and 403 SMMC-7721) and a human normal liver cell line (L02). $* * P<0.01$. GAPDH was used as 404 control. (B) LINC00844 expression in HepG2 and HCCLM9 cells after transfection with Lv405 LINC00844 was detected with RT-qPCR. **P<0.01. GAPDH was used as control. (C, D) 406 Proliferation was detected with the CCK-8 assay in HepG2 and HCCLM9 cells, $* * P<0.01$. (E, 407 F, G, and $\mathbf{H})$ The migration and invasion of HepG2 and HCCLM9 cells were examined with 408 transwell migration and invasion assays. ${ }^{*} P<0.01$. All experiments were performed in 409 triplicates.

410 Figure 5: NDRG1 was highly expressed in clinical HCC tissues and cell lines and negatively 411 correlated with LINC00844.

412 (A) Expression of NDRG1 was analysed in HCC from GEPIA. $* P<0.01$. (B) The relationship 413 between NDRG1 expression and pathological stage. (C) Kaplan-Meier analysis to detect the 414 correlation between NDRG1 expression and overall survival for HCC samples from GEPIA. ${ }^{*} P$ $415<0.05$. (D) qRT-PCR revealed the relative expression level of NDRG1 in 20 pairs of HCC 416 tissues and adjacent non-tumour tissues. $P<0.05$. GAPDH was used as control. (E) The 417 correlation between LINC00844 expression level and NDRG1 mRNA level in 20 HCC tissues. 418 The $\Delta \mathrm{CT}$ values were subjected to Pearson's correlation analysis. $\Delta \mathrm{CT}$ value was determined by 419 subtracting GAPDH Ct value from LINC00844 $\Delta \mathrm{Ct}$ value and NDRG1 Ct value. $P<0.05, \mathrm{R}^{2}=$ 420 0.3043. GAPDH was used as control. (F) Representative immunostaining to detect NDRG1 421 protein expression in HCC tissues and paired adjacent non-tumour tissues. (G) The changes in 422 NDRG1 mRNA and protein levels were examined in HepG2 and HCCLM9 cells overexpressing 
423 LINC00844. $* * P<0.01$. GAPDH was used as control. All results are obtained from at least 424 three independent experiments.

425 
Figure 1

Analysis of the expression of LINC00844 in TCGA and GEPIA databases.

(A) Heat map of the top five upregulated and top five downregulated IncRNAs. (B) Overview of LINC00844 expression in multiple tumour entities with a notable decrease in tumours as compared with that in normal tissues. The image is derived from GEPIA.

A

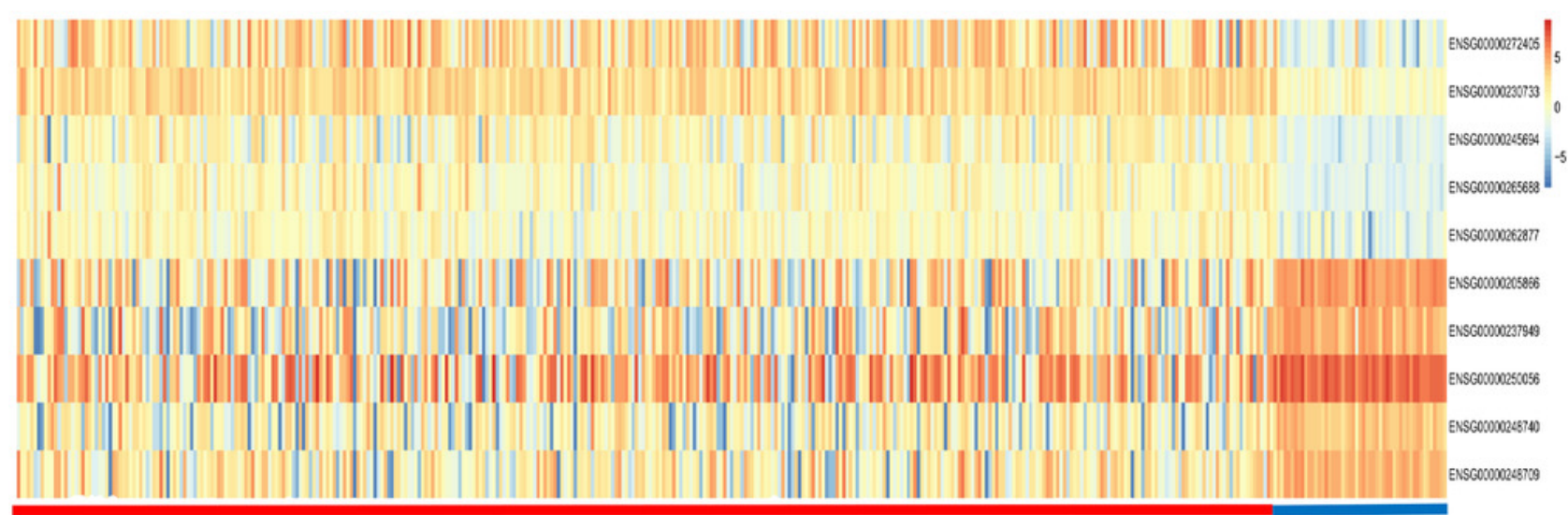

$\mathrm{B}$

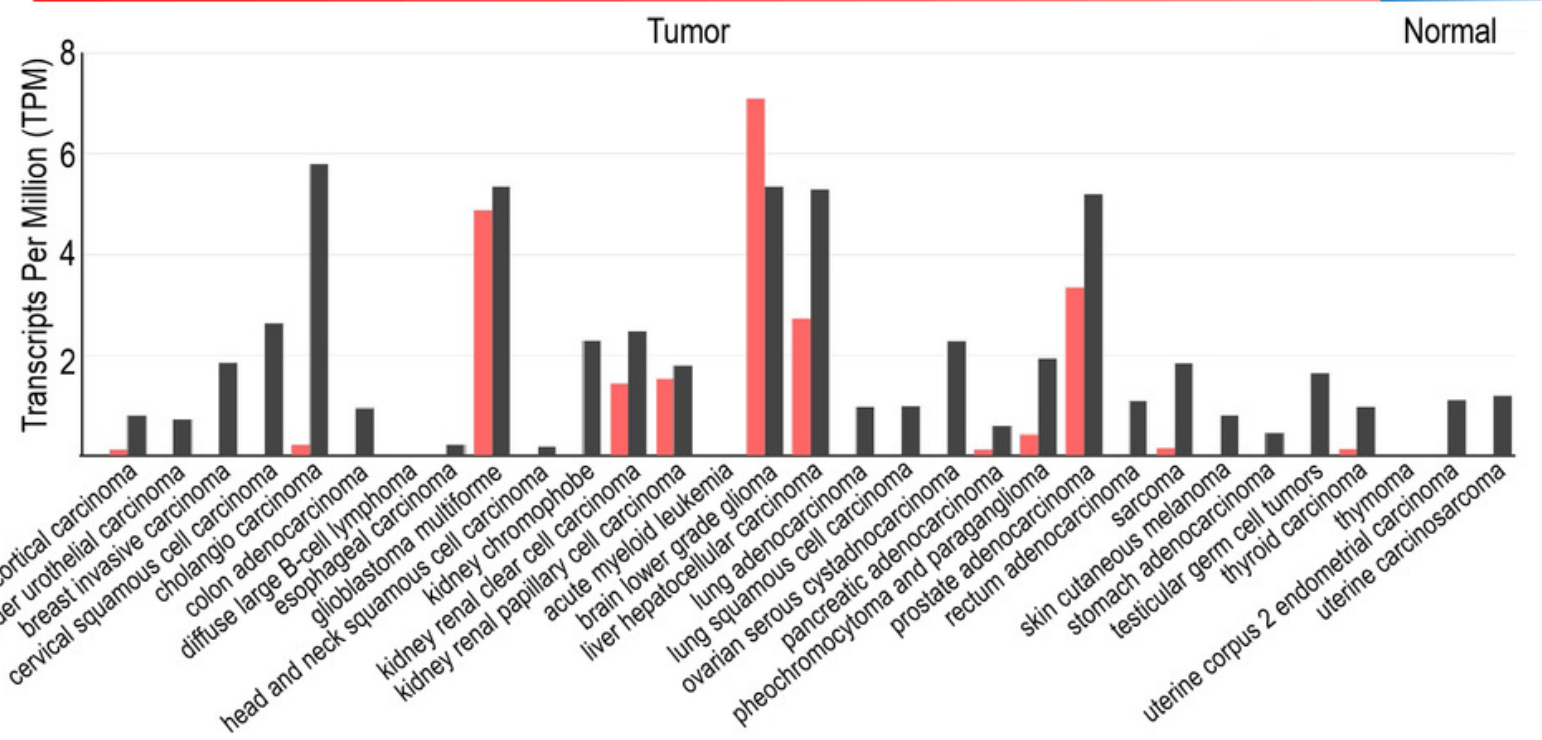




\section{Figure 2}

LINC00844 is significantly downregulated in HCC.

(A) Expression of LINC00844 was analysed in HCC from GEPIA. $* P<0.01$. (B) The relationship between the expression of LINC00844 and HCC pathological stage from GEPIA. $* P<0.05$. (C and D) Kaplan-Meier analysis of the correlation between LINC00844 expression and disease-free survival and overall survival in GEPIA. $P>0.05$. (E) ROC curve of LINC00844 in HCC derived from the original data from TCGA database. $P<0.01, A U C=0.854$. ( $F$ and G) Expression and fold change of LINC00844 in 40 pairs of HCC tissues and adjacent nontumour tissues. Downregulation of LINC00844 (> twofold change) expression was detected in $87.5 \%$ (35/40) of HCC. ${ }^{* * P}<0.01$. GAPDH was used as control. All results are obtained from at least three independent experiments. 
A

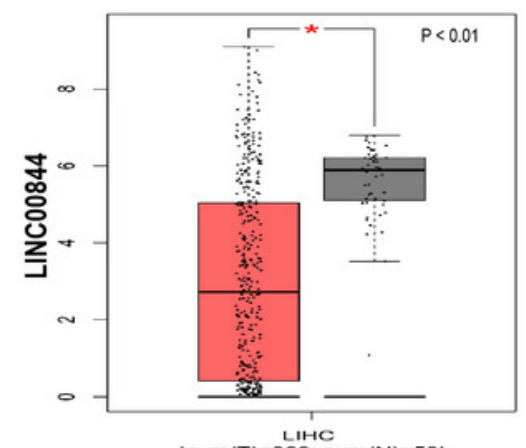

D

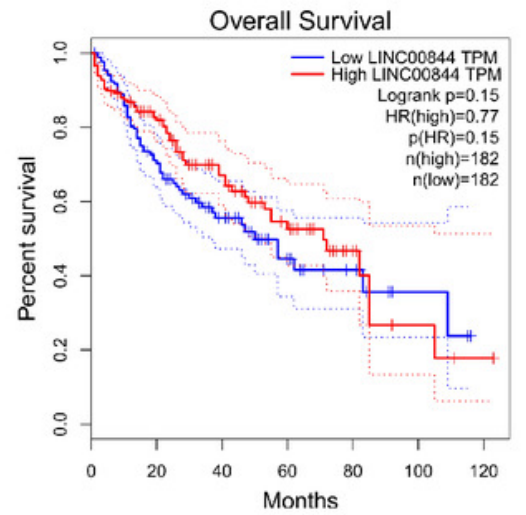

B

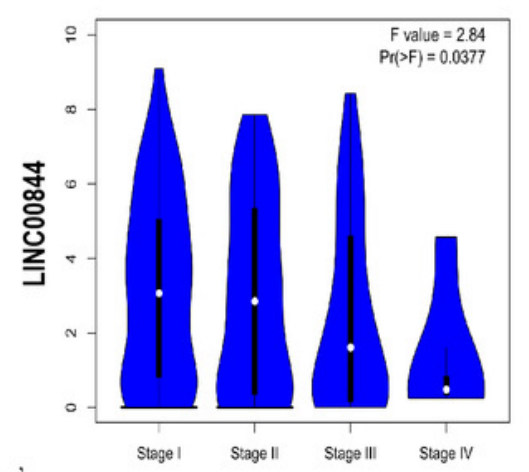

i

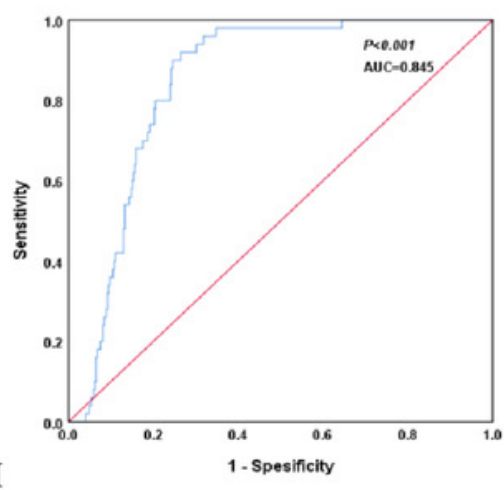

C
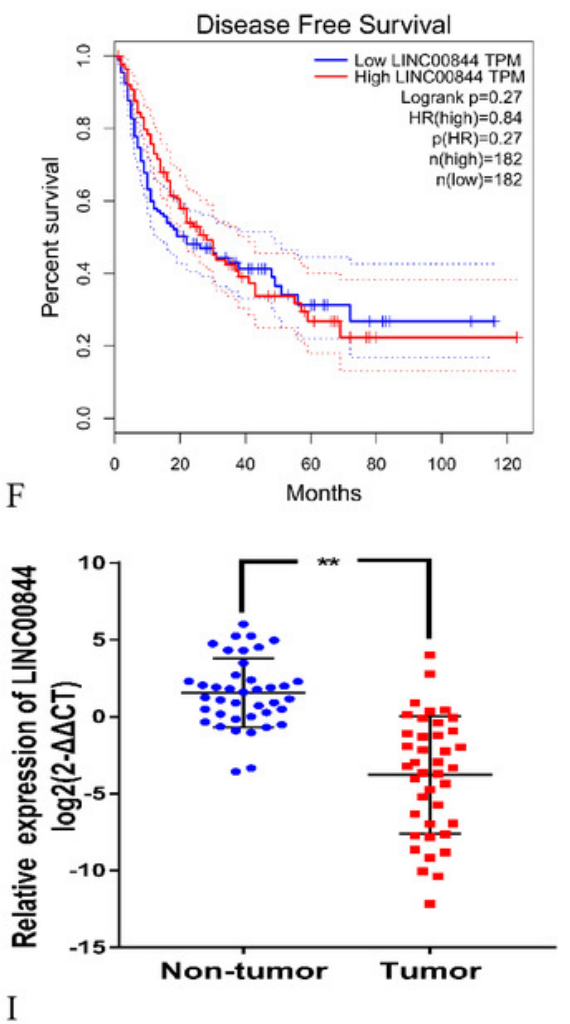

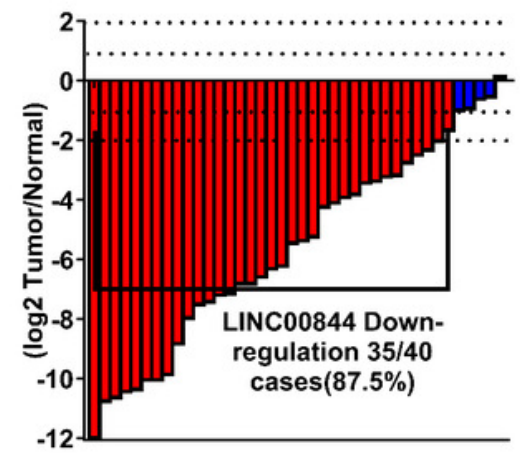


Figure 3

Correlation between LINC00844 downregulation and clinicopathologic features in 254 HCC samples and 50 normal liver tissues based on the original data from TCGA.

(A, C, E, and F) The relationship between LINC00844 expression and sex (Male versus Female, $P<0.001$ ), histological grade (G1 + G2 versus $\mathrm{G} 3+\mathrm{G} 4, P<0.001$ ), advanced American Joint Committee on Cancer (AJCC) stage (I + II versus III + IV, P=0.0399), and vascular invasion (None versus Micro versus Macro, $P=0.006$ ). (B and $\mathbf{D}$ ) The relationship between the expression of LINC00844 and ethnicity (Asian versus White, $P=0.5361$ ) and T classification of TNM stage (T1 $+\mathrm{T} 2$ versus $\mathrm{T} 3+\mathrm{T} 4, P=0.1366)$.

A

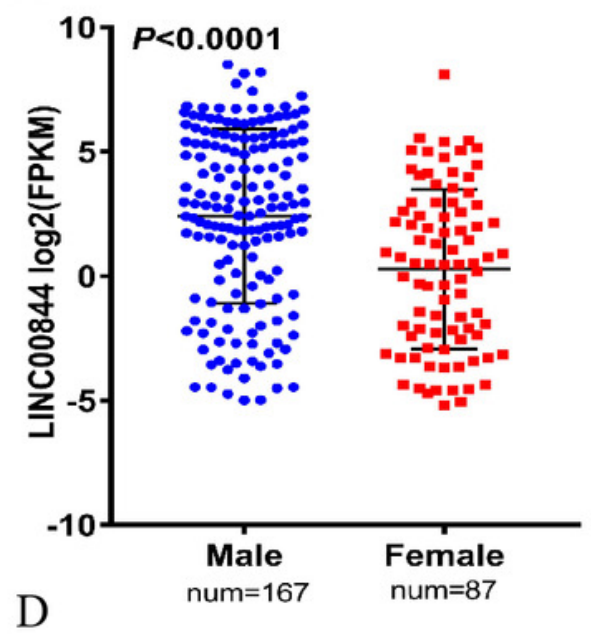

$\mathrm{D}$

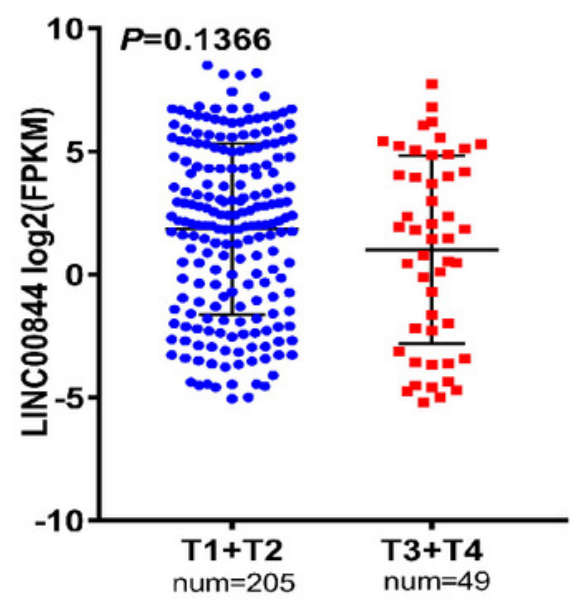

B

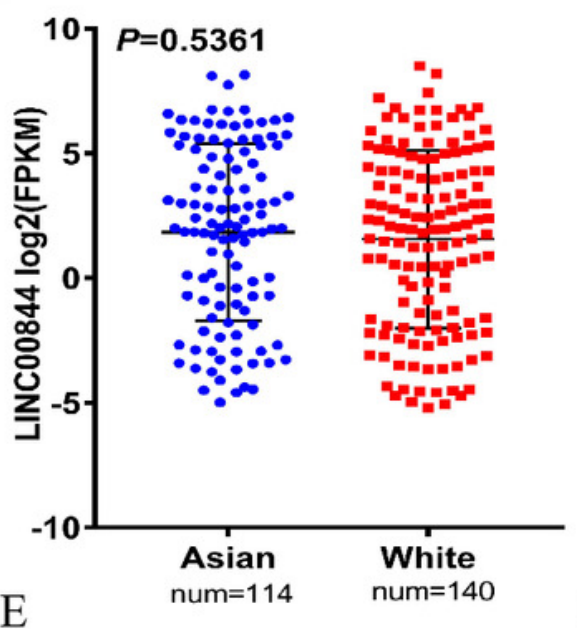

$\mathrm{E}$

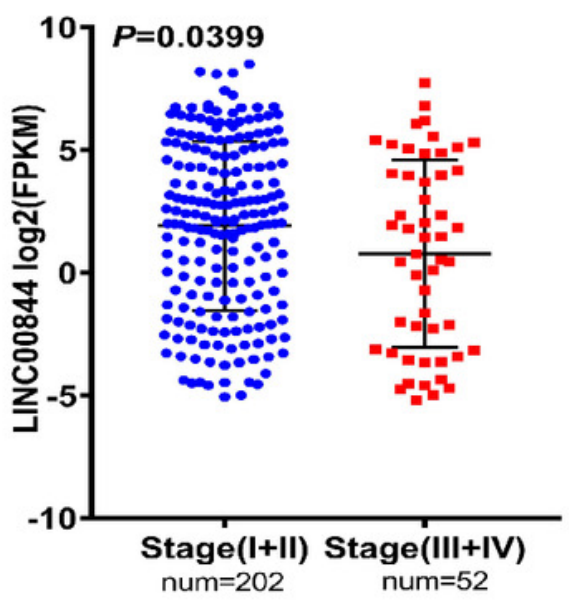

C
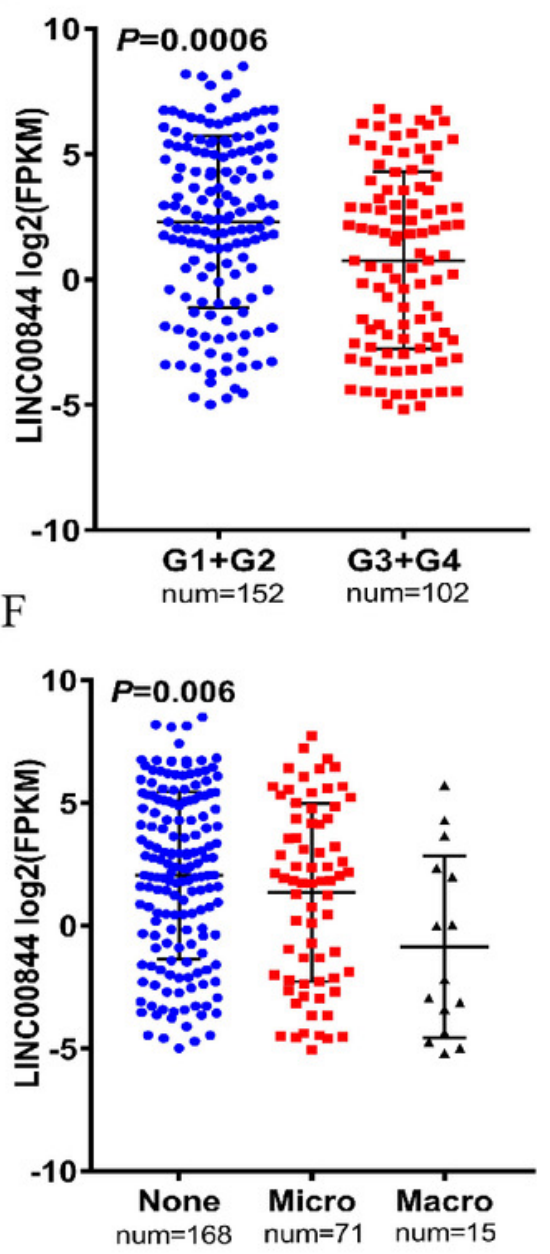


\section{Figure 4}

Upregulation in LINC00844 expression represses the proliferation, migration, and invasion of HepG2 and HCCLM9 cells.

(A) The relative expression of LINC00844 in HCC cell lines (HepG2, SK-Hep1, HCCLM9, and SMMC-7721) and a human normal liver cell line (L02). ${ }^{* * P}<0.01$. GAPDH was used as control. (B) LINC00844 expression in HepG2 and HCCLM9 cells after transfection with LVLINC00844 was detected with RT-qPCR. ${ }^{* * P}<0.01$. GAPDH was used as control. (C, D) Proliferation was detected with the CCK-8 assay in HepG2 and HCCLM9 cells, $* * P<0.01$. (E, F, G, and $\mathbf{H}$ ) The migration and invasion of HepG2 and HCCLM9 cells were examined with transwell migration and invasion assays. $* * P<0.01$. All experiments were performed in triplicates. 
A

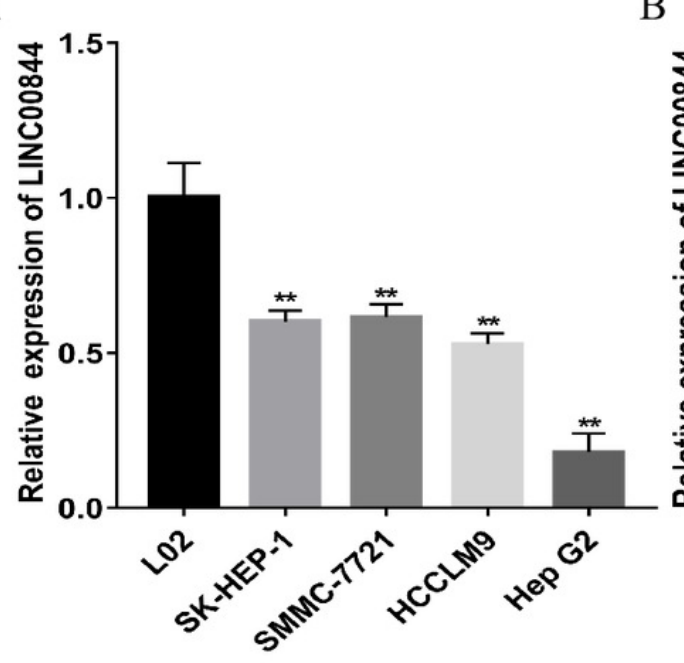

$\mathrm{C}$

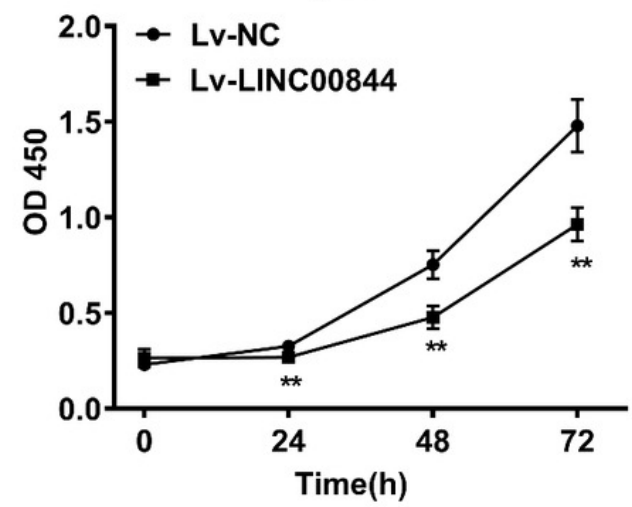

E

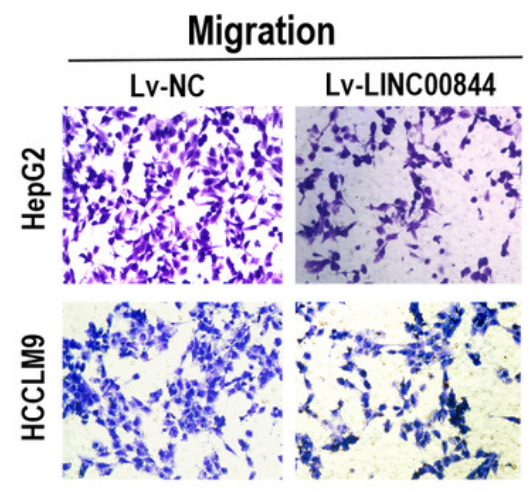

G

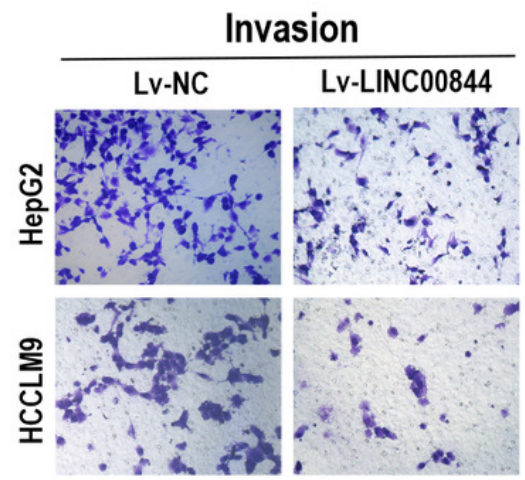

B

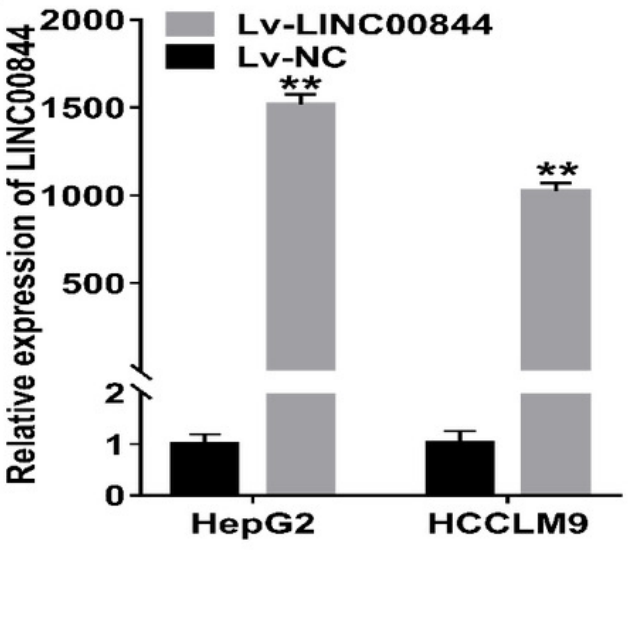

$\mathrm{D}$

HCCLM9

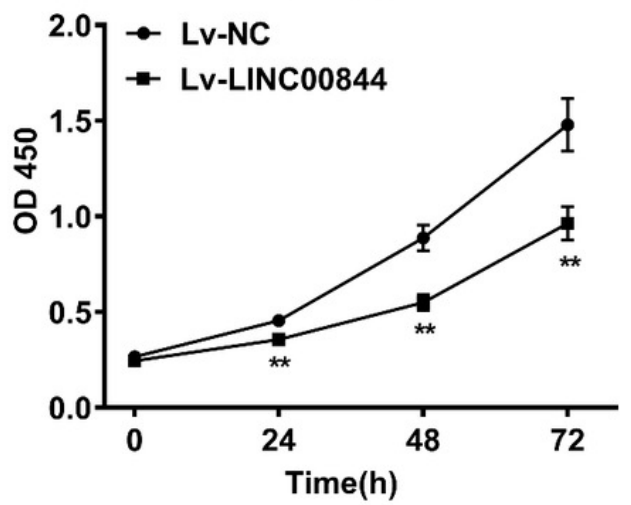

$\mathrm{F}$

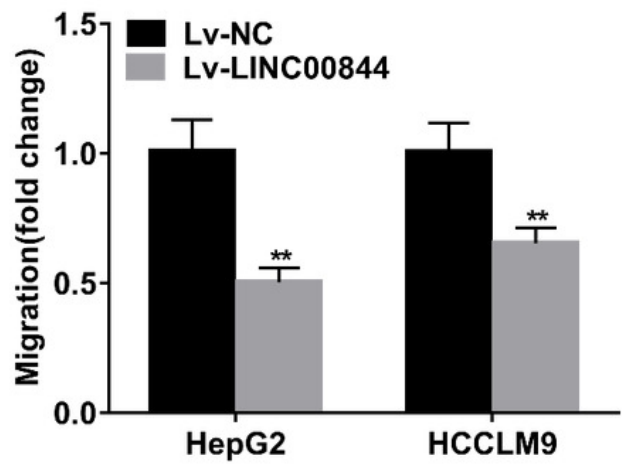

$\mathrm{H}$

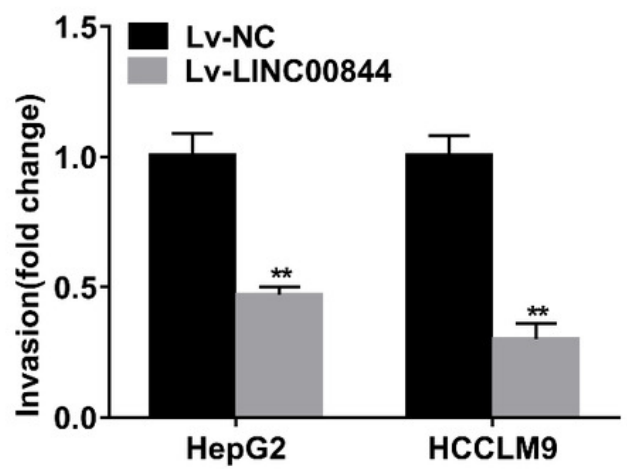




\section{Figure 5}

NDRG1 was highly expressed in clinical HCC tissues and cell lines and negatively correlated with LINC00844.

(A) Expression of NDRG1 was analysed in HCC from GEPIA. $* \boldsymbol{P}<0.01$. (B) The relationship between NDRG1 expression and pathological stage. (C) Kaplan-Meier analysis to detect the correlation between NDRG1 expression and overall survival for HCC samples from GEPIA. $* P<0.05$. (D) $q$ RT-PCR revealed the relative expression level of NDRG1 in $\mathbf{2 0}$ pairs of HCC tissues and adjacent non-tumour tissues. $P<0.05$. GAPDH was used as control. (E) The correlation between LINC00844 expression level and NDRG1 mRNA level in 20 HCC tissues. The $\triangle C T$ values were subjected to Pearson's correlation analysis. $\Delta C T$ value was determined by subtracting GAPDH Ct value from LINC00844 $\Delta$ Ct value and NDRG1

Ct value. $P<0.05, R^{2}=0.3043$. GAPDH was used as control. (F) Representative immunostaining to detect NDRG1 protein expression in HCC tissues and paired adjacent non-tumour tissues. (G) The changes in NDRG1 mRNA and protein levels were examined in HepG2 and HCCLM9 cells overexpressing LINC00844. $* * P<0.01$. GAPDH was used as control. All results are obtained from at least three independent experiments. 
A

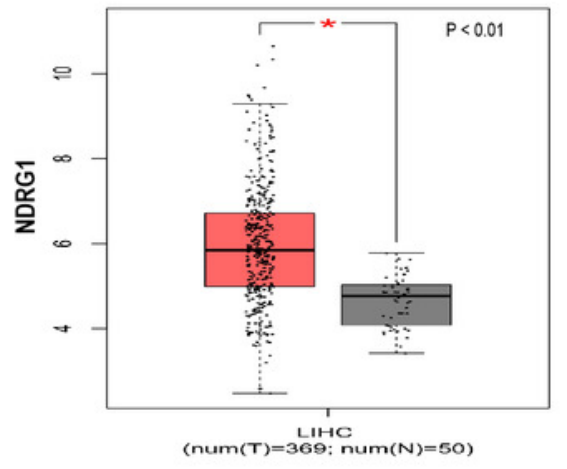

D

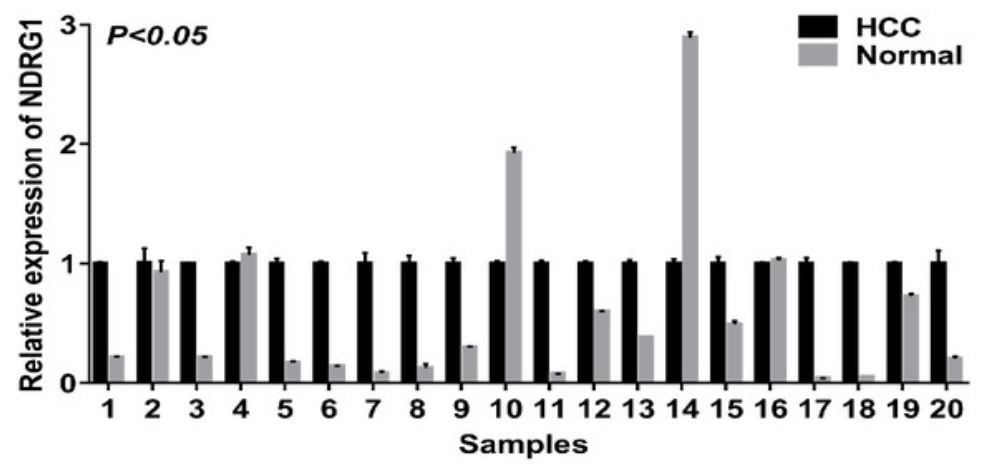

B

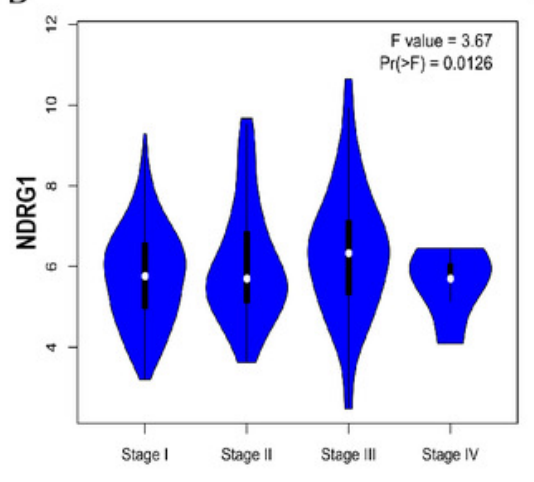

$\mathrm{C}$

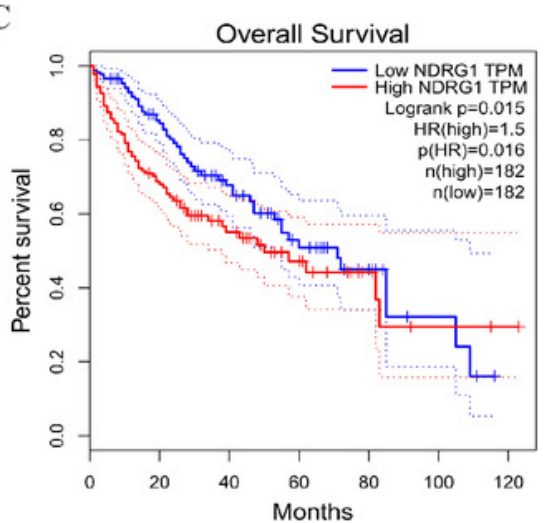

E

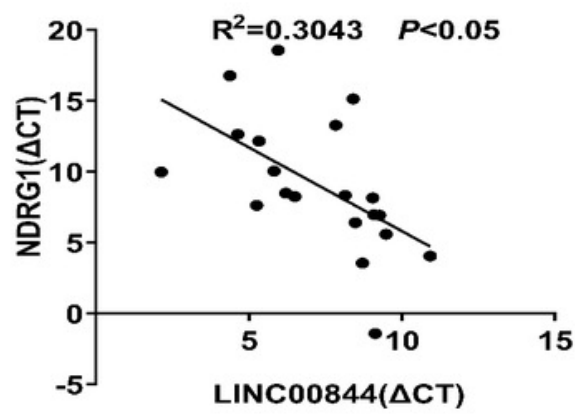

F

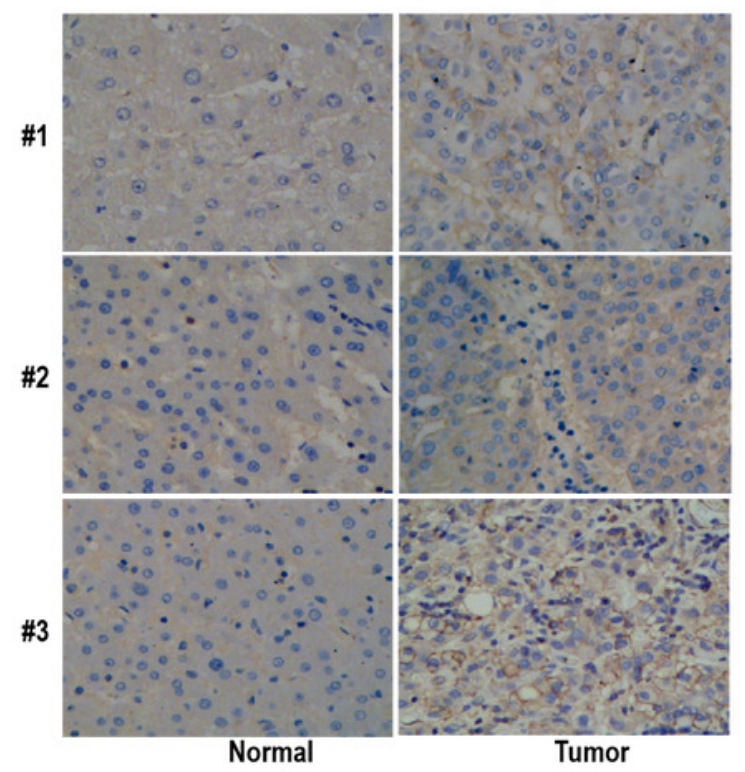

G
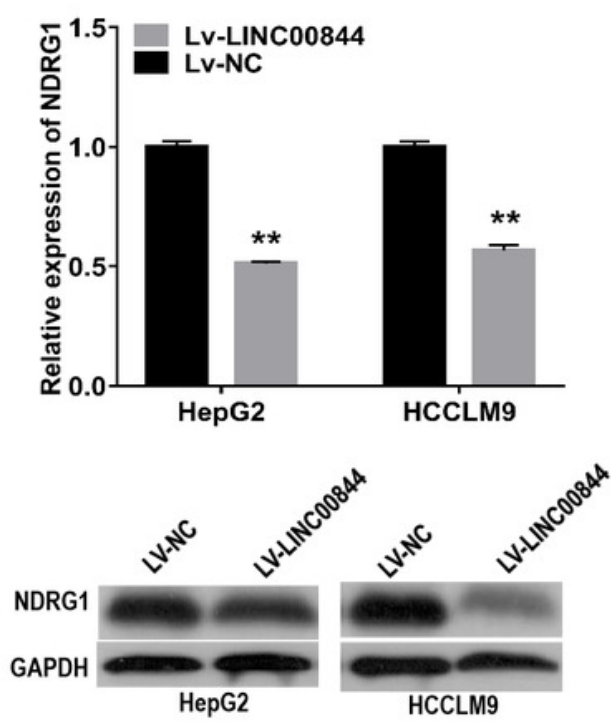
Table $\mathbf{1}$ (on next page)

All primers sequences 
1

2

3

4

5

6

7

8

9

10

11

12

\begin{tabular}{cl}
\hline Gene & \multicolumn{1}{c}{ Primer sequence $\left(5^{\prime}-3^{\prime}\right)$} \\
\hline \multirow{2}{*}{ LINC00844 } & CTTGATGCAGTCTGATAGGAGGAT \\
& TCTGCCATACTGTTTCTGGTTCA \\
NDRG1 & TACCGCCAGCACATTGTGAA \\
& GCCACAGTCCGCCATCTT \\
GAPDH & GGAAGCTTGTCATCAATGGAAATC \\
& TGATGACCCTTTTGGCTCCC \\
\hline
\end{tabular}

LINC00844,Long intergenic non-protein coding RNA 844; NDRG1,N-myc downstream regulated 1; GAPDH,glyceraldehyde-3-phosphate dehydrogenase. 


\section{Table 2 (on next page)}

the top 5 upregulated IncRNAs and the top 5 downregulated IncRNA 


\begin{tabular}{cccccc}
\hline Regulation & Ensemble ID & Gene symbol & Log2FC & $P$-value & FDR \\
\hline \multirow{5}{*}{ Up-regulation } & ENSG00000272405 & AL365181.3 & 3.50 & $4.58 \mathrm{E}-08$ & $1.71 \mathrm{E}-07$ \\
& ENSG00000230733 & AC092171.2 & 2.59 & $2.81 \mathrm{E}-23$ & $9.38 \mathrm{E}-22$ \\
& ENSG00000265688 & MAFG-AS1 & 2.23 & $9.61 \mathrm{E}-15$ & $9.43 \mathrm{E}-14$ \\
& ENSG00000262877 & AC110285.2 & 2.07 & $1.30 \mathrm{E}-13$ & $1.09 \mathrm{E}-12$ \\
\hline \multirow{5}{*}{ Down-regulation } & ENSG00000205866 & FAM99A & -4.61 & $3.37 \mathrm{E}-39$ & $5.89 \mathrm{E}-37$ \\
& ENSG000000237949 & LINC00844 & -4.27 & $1.73 \mathrm{E}-32$ & $1.89 \mathrm{E}-30$ \\
& ENSG00000248740 & LINC02428 & -3.75 & $1.14 \mathrm{E}-33$ & $1.41 \mathrm{E}-31$ \\
& ENSG00000248709 & AC008549.1 & -3.59 & $4.77 \mathrm{E}-31$ & $4.01 \mathrm{E}-29$ \\
\hline
\end{tabular}

FC,fold change; FDR,false discovery rate 
Table 3(on next page)

The correlation between LINC00844 expression and the clinicopathological features of patients with HCC. 


\begin{tabular}{|c|c|c|c|c|}
\hline \multirow{2}{*}{ Clinicopathological parameters } & \multicolumn{4}{|c|}{ LINC00844 } \\
\hline & $\mathrm{N}$ & Mean+SEM & $\mathrm{T}$ & $P$-value \\
\hline \multicolumn{5}{|l|}{ Tissues } \\
\hline Normal liver & 50 & $4.953 \pm 0.154$ & \multirow{2}{*}{6.437} & \multirow{2}{*}{$<0.000$} \\
\hline $\mathrm{HCC}$ & 345 & $1.803 \pm 0.185$ & & \\
\hline \multicolumn{5}{|l|}{ Sex } \\
\hline Male & 167 & $2.411 \pm 0.271$ & \multirow{2}{*}{4.710} & \multirow{2}{*}{$<0.000$} \\
\hline Female & 87 & $0.288 \pm 0.344$ & & \\
\hline \multicolumn{5}{|l|}{ Race } \\
\hline Asian & 114 & $1.837 \pm 0.332$ & \multirow{2}{*}{0.620} & \multirow{2}{*}{0.536} \\
\hline White & 140 & $1.559 \pm 0.301$ & & \\
\hline \multicolumn{5}{|l|}{$\mathrm{T}$ (tumor) } \\
\hline $\mathrm{T} 1+\mathrm{T} 2$ & 205 & $1.846 \pm 0.242$ & \multirow{2}{*}{1.493} & \multirow{2}{*}{0.137} \\
\hline $\mathrm{T} 3+\mathrm{T} 4$ & 49 & $1.005 \pm 0.546$ & & \\
\hline \multicolumn{5}{|l|}{ Stage } \\
\hline $\mathrm{I}+\mathrm{II}$ & 202 & $1.196 \pm 0.243$ & \multirow{2}{*}{2.066} & \multirow{2}{*}{0.040} \\
\hline $\mathrm{III}+\mathrm{IV}$ & 52 & $0.783 \pm 0.530$ & & \\
\hline \multicolumn{5}{|l|}{ Histological grade } \\
\hline $\mathrm{G} 1+\mathrm{G} 2$ & 152 & $2.305 \pm 0.279$ & \multirow{2}{*}{3.481} & \multirow{2}{*}{0.001} \\
\hline $\mathrm{G} 3+\mathrm{G} 4$ & 102 & $0.758 \pm 0.350$ & & \\
\hline \multicolumn{5}{|l|}{ Vascular invasion } \\
\hline None & 168 & $2.050 \pm 0.264$ & \multirow{3}{*}{$\mathrm{F}=5.215$} & \multirow{3}{*}{0.006} \\
\hline Micro & 71 & $1.352 \pm 0.430$ & & \\
\hline Macro & 15 & $-0.856 \pm 0.954$ & & \\
\hline
\end{tabular}




\section{Table 4 (on next page)}

The relationships of LINC00844 expression with clinicopathological parameters in HCC based on $40 \mathrm{HCC}$ patients. 
1 Table4 The relationships of LINC00844 expression with clinicopathological parameters in $2 \mathrm{HCC}$

\begin{tabular}{|c|c|c|c|}
\hline \multirow{2}{*}{ Variable } & \multirow{2}{*}{$\begin{array}{c}\text { Number of } \\
\text { cases }\end{array}$} & LINC01554 expression & \multirow{2}{*}{$P$-value } \\
\hline & & $\operatorname{High}(\mathrm{N}=20)$ & \\
\hline
\end{tabular}

Gender

$\begin{array}{lllll}\text { Male } & 33 & 14 & 19 & 0.0915\end{array}$

Female

7

6

1

Age(year)

$\begin{array}{lcccc}\leq 50 & 8 & 3 & 5 & 0.6948 \\ >50 & 32 & 17 & 15 & \end{array}$

HBV infection

Negative

Positive

Serum

$\operatorname{AFP}(\mathrm{ng} / \mathrm{mL})$

$\leq 8.78$

$>8.78$

Pathological stage

$$
\begin{aligned}
& \text { I }+ \text { II } \\
& \text { III }+ \text { IV }
\end{aligned}
$$

16

15

11

3

0.4506

14

17

Cirrhosis

No

11

7

4 
$\begin{array}{lll}\text { Yes } & 29 & 13\end{array}$

PVTT

No 35

35

15

20

0.0471

Yes

5

5

0

TNM(AJCC)

$\mathrm{I}+\mathrm{II}$

26

9

17

0.0187

III + IV

14

11

3

3 PVTT portal vein tumor thrombus

4 AJCC American Joint Committee on Cancer

$5 \quad * P<0.05$ 